\title{
Cutaneous necrotizing vasculitis as a first manifestation of antiphospholipid syndrome with catastrophic development
}

\author{
Lucia Todaro, ${ }^{1}$ Angela Luppino, ${ }^{1}$ Renata Carra, ${ }^{1}$ Aldo Nocchiero, ${ }^{2}$ Anna Maria Marson, ${ }^{2}$ Egidio Genovese, ${ }^{3}$ \\ Federico Ragazzoni, ${ }^{1}$ Roberto Quadri ${ }^{1}$ \\ ${ }^{1}$ Division of Internal Medicine, Ospedale Civico (ASLTO4), Chivasso (TO); ${ }^{2}$ Division of Neurology, Ospedale Civico, \\ Chivasso (ASLTO4), Chivasso (TO); ${ }^{3}$ Neuroradiology Unit, Clinica Eporediese, Ivrea (TO), Italy
}

\begin{abstract}
We describe a case of anti-phospholipid syndrome, with catastrophic development and unusual brain involvement, in which the first manifestation was necrotizing cutaneous vasculitis. A 58-year-old woman presented with fever, gangrene of her lower limbs and ischemic lesions of her arms and hands. Laboratory results showed normal white cell count and normal procalcitonin value. The vascular ultrasound study of the lower limbs did not show any thrombosis or stenosis. A chest-abdomen computed tomography scan showed inflammatory thickening of the lung. A month after admission she developed confusion, weakness and aphasia. Magnetic resonance imaging of the brain showed hyperintense bilateral symmetrical lesions in the frontal and cerebellar regions. Anti-cardiolipin antibodies and lupus anticoagulant were negative, whereas anti- $\beta 2$ glycoprotein 1 were in high titer. She was started on steroids, and subsequently warfarin, acetylsalicylic acid and hydroxychloroquine, with progressive improvement of her clinical status.
\end{abstract}

\section{Introduction}

Anti-phospholipid syndrome (APS) is an autoimmune disease characterized by venous and arterial thrombosis, miscarriage or preterm delivery, and persistent positive laboratory tests for antiphospholipid antibodies (aPLs); aPLS include lupus anticoagulant (LAC) and/or anti-cardiolipin antibodies (ACA) [immunoglobulin (Ig) G or IgM] and/or anti- $\beta 2$ glycoprotein 1 (anti- $\beta 2 \mathrm{GPI})\left(\mathrm{IgG}\right.$ or IgM). ${ }^{1,2}$ Catastrophic

Correspondence: Lucia Todaro, Division of Internal Medicine, Ospedale Civico, corso Galileo Ferraris 3, 10034 Chivasso (TO), Italy.

Tel./Fax: +39.011.9176228

E-mail: federico.ragazzoni@yahoo.it

Key words: Vasculitis; antiphospholipid syndrome; cutaneous necrosis.

Conflict of interest: the authors declare no potential conflict of interest.

Received for publication: 6 March 2016.

Revision received: 17 October 2016.

Accepted for publication: 21 October 2016.

This work is licensed under a Creative Commons Attribution NonCommercial 4.0 License (CC BY-NC 4.0).

(C) Copyright L. Todaro et al., 2017

Licensee PAGEPress, Italy

Italian Journal of Medicine 2017; 11:215-217

doi:10.4081/itjm.2017.710
APS (CAPS) is the most severe form of APS. It is an acute biological process that leads to multiple organ failure in a short time, due to microthrombosis of small vessels. ${ }^{3,4}$ It occurs in less than $1 \%$ of APS cases, but early diagnosis is very important because of high mortality and morbility. ${ }^{5}$ Other thrombotic microangiopathic conditions need to be considered in the differential diagnosis: thrombotic thrombocytopenic purpura, hemolytic uremic syndrome, hemolysis, elevated liver enzymes, low platelets syndrome. ${ }^{6}$

\section{Case Report}

A 58-year-old Polish woman was admitted with a suspected septic condition. She presented with gangrene of her legs, necrosis of the $2^{\text {nd }}$ and $3^{\text {rd }}$ toe of her left foot, ischemic lesions of her arms and hands, fever and dyspnea. Reactive $\mathrm{C}$ protein was elevated, while procalcitonin, renal function, platelet count and liver enzymes were normal; D-dimer was 2.49 (reference $0-0.5 \mathrm{ug} / \mathrm{mL}$ ). The vascular ultrasound study of her lower limbs did not show any thrombosis or stenosis. Echocardiogram was normal and blood cultures were sterile. Exudate collected from the gangrenous lesions was positive for Pseudomonas aeruginosa. The patient was treated with antibiotics, morphine and low molecular weight heparin (enoxaparin 4000 $\mathrm{UI} \times 2 /$ die). First hypothesis was of an autoimmune or paraneoplastic cutaneous vasculitis, but anti-nuclear antibodies, anti-neutrophil cytoplasmic antibodies, anti-extractable nuclear antigen antibodies cryoglobulins, C3, C4 and onco-hematological markers were 
in the normal range. The fever disappeared and skin lesions improved after debridement, but the patient maintained a condition of mild type 1 respiratory failure; chest-abdomen computed tomography (CT) scan showed inflammatory thickening of the lung. Around one month after admission she developed confusion, weakness and aphasia. Brain magnetic resonance imaging showed hyperintense bilateral and symmetrical lesions in the frontal and cerebellar regions (Figures 1 and 2). A total body angio-CT was also performed, that showed multiple cerebral ischemic areas and tight stenosis at the origin of the celiac trunk. Liquor examination was normal. She was started on acetylsalicylic acid $100 \mathrm{mg} / \mathrm{die}$ and clopidogrel $75 \mathrm{mg} / \mathrm{die}$. The presence of aPLS was tested: ACA and LAC were negative, whereas anti- $\beta 2$ GPI antibodies were found in high titer $(100 \mathrm{U} / \mathrm{mL}$, normal values: 1.0-8.0). These findings allowed us to formulate the diagnosis of CAPS, and she was started on high-dosage steroids (prednisone $75 \mathrm{mg} / \mathrm{die}$ ); after 15-day prednisone was replaced with hydroxychloroquine (200 $\mathrm{mg}$ bid), clopidogrel and warfarin, obtaining a progressive improvement of the whole clinical picture, as well as remission of the neurological symptoms. The patient subsequently left Italy and we were therefore unable to repeat the laboratory tests.

\section{Discussion}

APS is a debilitating syndrome, especially because of neurological damage; in fact a cognitive dysfunction has been recorded in more than $40 \%$ of patients, and stroke or transient ischemic attack (TIA) occurred in more than $20 \%$ of cases. ${ }^{7}$ Thrombotic neurological manifestations include ischemic stroke, TIA, acute is-

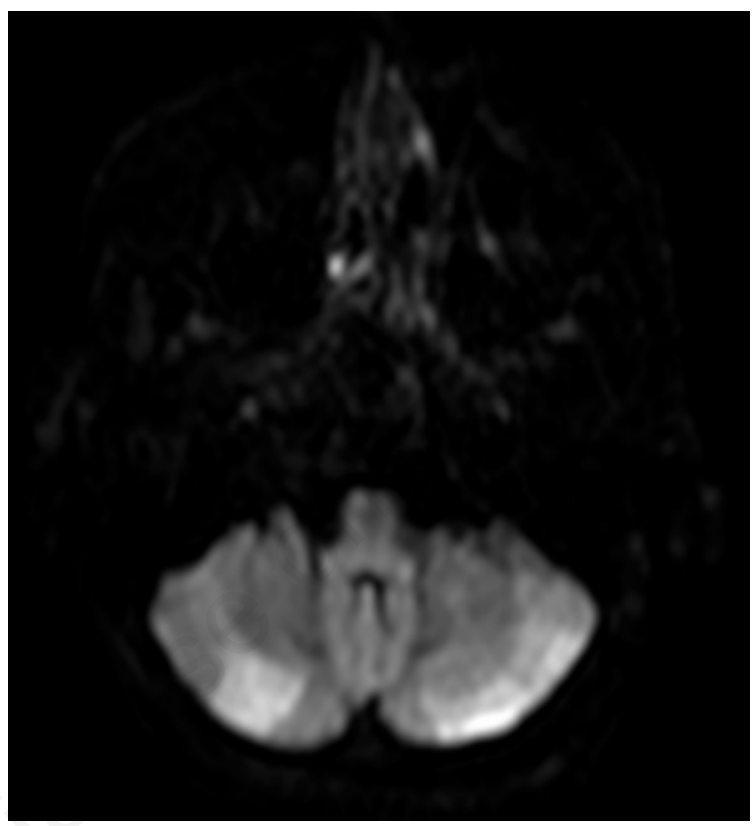

Figure 1. Diffusion weighted imaging on magnetic resonance imaging, which reveals the presence of a restriction of the apparent diffusion coefficient in the cerebellar regions and bilaterally in the parietal lobes.

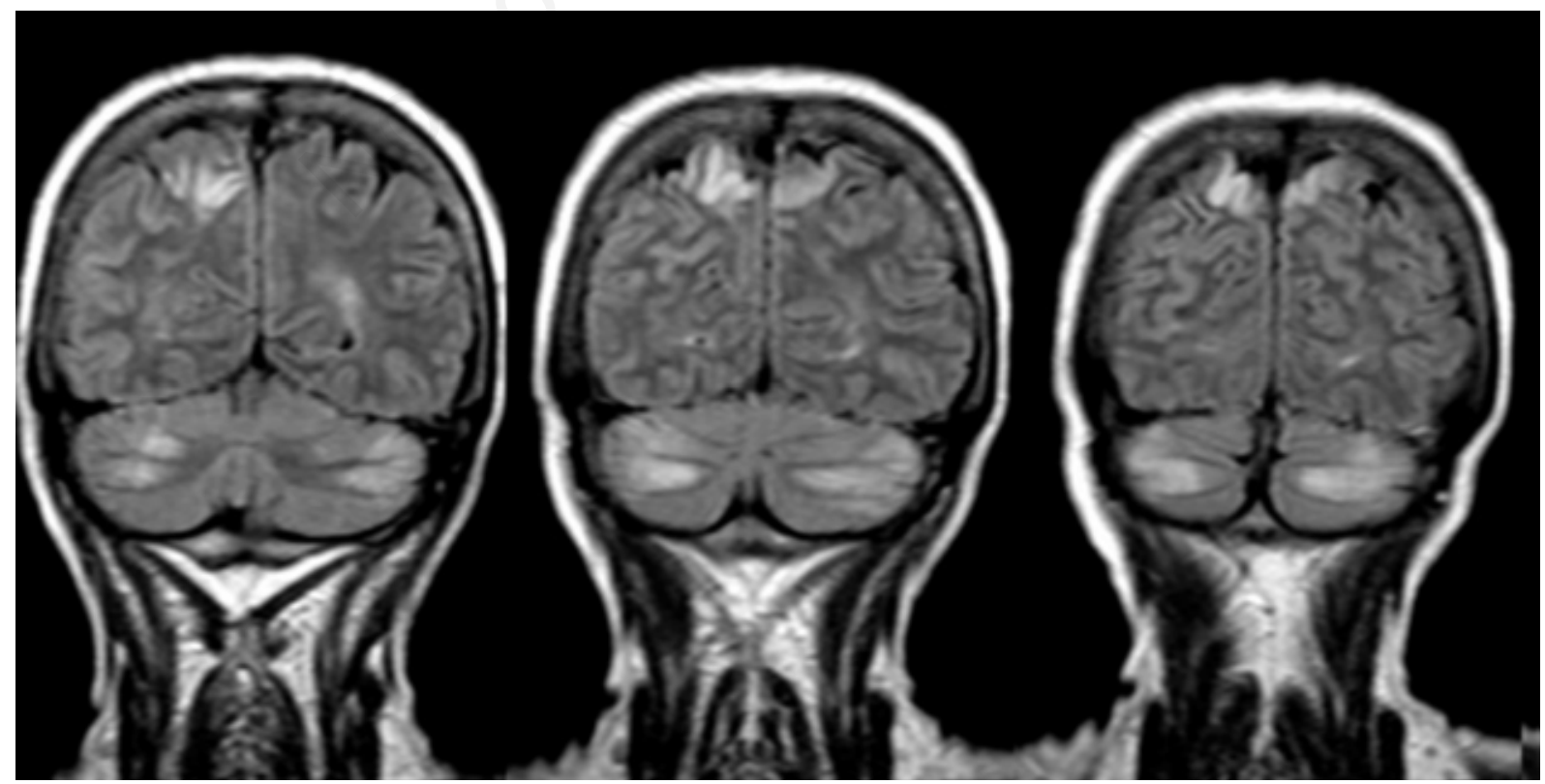

Figure 2. Fluid attenuation inversion recovery sequence on magnetic resonance imaging, which reveals the presence of almost symmetrical hyperintense bilateral lesions in the frontal and cerebellar regions. 
chemic encephalopathy, spinal cord stroke, cerebral venous thrombosis; the non-thrombotic neurological manifestations, described in up to $40 \%$ of patients with elevated aPLS are headache, transverse myelitis, sensorineural hearing loss, seizures, multiple sclerosis-like lesions, peripheral neuropathy, dementia, idiopathic intracranial hypertension and chorea. ${ }^{8}$ Brain involvement in CAPS occurs in $55.9 \%$ of cases and the most frequent cerebral manifestations are encephalopathy $(40.2 \%)$, stroke $(35.2 \%)$, seizures $(14.6 \%)$, headache $(8.5 \%)$ and coma $(6.1 \%)$, according to the CAPS registry. ${ }^{5}$ The peculiarity of this case is the symmetry of the cerebral ischemic lesions and the development in outbreaks. Regarding the cutaneous manifestation of APS, the most frequent is livedo reticularis that occurs in a quarter of all patients; the presence of leg ulcers ${ }^{6}$ is more unusual ( $<10 \%$ of cases). In CAPS the most frequent skin complications are livedo reticularis (42.3\%), skin necrosis $(25.2 \%)$, ulcers $(23.5 \%)$ and digital ischemia $(10 \%){ }^{5}$

In our patient, skin and digital necrosis was the onset manifestation, and the infection could be the trigger for CAPS.

\section{Conclusions}

This case teaches us to consider immediately the diagnosis of APS in a patient presenting with ischemic skin lesions and necrosis of the toes and/or fingers.
The severity of clinical manifestations, in particular regards of neurological involvement, and the clinical evolution rapidly impose an early diagnosis and treatment in order to reduce the probability of occurrence of CAPS or to improve the prognosis of this syndrome.

\section{References}

1. Lim W. Antiphospholipid syndrome. Hematology 2013;1:675-80.

2. Miyakis S, Lockshin MD, Atsumi T, et al. International consensus statement on an update of the classifiction criteria for definite antiphospholipid syndrome (APS). J Thromb Haemost 2006;2:295-306.

3. Asherson RA. The catastrophic antiphospholipid syndrome. J Rheumatol 1992;19:508-12.

4. Merril JT, Asherson RA. Catastrophic antiphospholipid syndrome. Nat Clin Pract Rheumatol 2006;2:81-9.

5. Cervera R, Rodriguez-Pintò I, Colafrancesco S, et al. $14^{\text {th }}$ International congress on antiphospholipid antibodies task force report on catastrophic antiphospholipid syndrome. Autoimmunity Rev 2014;13:699-707.

6. Erkan D, Espinosa G, Cervera R. Catastrophic antiphospholipid syndrome: updated diagnostic algorithms. Autoimmunity Rev 2010;10:74-9.

7. Ruiz-Irastorza G, Crowther M, Branch W, et al. Antiphospholipid syndrome. Lancet 2010;376:1498-509.

8. Carecchio M, Cantello R, Comi C. Revisiting the molecular mechanism of neurological manifestations in antiphospholipid syndrome: beyond vascular damage. J Immunol Res 2014;1-9. 